NOTICE: this is the author's version of a work that was accepted for publication in Systems \& Control Letters. Changes resulting from the publishing process, such as peer review, editing, corrections, structural formatting, and other quality control mechanisms may not be reflected in this document. Changes may have been made to this work since it was submitted for publication. A definitive version was subsequently published in Systems \& Control Letters [VOL54, ISSUE7, 2005] http://dx.doi.org/10.1016/j.sysconle.2004.11.008 


\title{
Employing the algebraic Riccati equation for a parametrization of the solutions of the finite-horizon LQ problem: the discrete-time case*
}

\author{
Augusto Ferrante Lorenzo Ntogramatzidis* $^{\star}$ \\ ${ }^{\ddagger}$ Dipartimento di Elettronica e Informatica \\ Università di Padova, via Gradenigo, 6/B - 35131 Padova, Italy \\ tel: +390498277681 \\ augusto@dei.unipd.it \\ ${ }^{\star}$ Dipartimento di Elettronica, Informatica e Sistemistica \\ Università di Bologna, viale Risorgimento, 2 - 40136 Bologna, Italy \\ tel: +39051 2093046 - fax: +39051 2093073 \\ lntogramatzidis@deis.unibo.it
}

February 13, 2006

\begin{abstract}
In this paper a new methodology is developed for the closed-form solution of a generalized version of the finite-horizon linear-quadratic regulator problem for LTI discrete-time systems. The problem considered herein encompasses the classical version of the LQ problem with assigned initial state and weighted terminal state, as well as the so-called fixed-end point version, in which both the initial and the terminal states are sharply assigned. The present approach is based on a parametrization of all the solutions of the extended symplectic system. In this way, closed-form expressions for the optimal state trajectory and control law may be determined in terms of the boundary conditions. By taking advantage of standard software routines for the solution of the algebraic Riccati and Stein equations, our results lead to a simple and computationally attractive approach for the solution of the considered optimal control problem without the need of iterating the Riccati difference equation.
\end{abstract}

Keywords: discrete-time systems, quadratic cost function, finite-horizon LQ problem, extended symplectic system.

\footnotetext{
${ }^{*}$ Partially supported by the ministry of higher education of Italy (MIUR), under project Identification and Control of Industrial Systems.
} 


\section{Introduction}

The finite-horizon linear quadratic regulator (LQR) is a well-known and deeply investigated problem in control theory. The solution of this optimization problem is obtained in the literature by resorting to the solution of a suitable Riccati differential/difference equation (see for example [1], [8] and [11]), which is very demanding from a computational point of view. To lighten this computational burden, in [5] a new approach has been presented for the solution, in the continuous-time case, of the finite-horizon LQ problem, when both the initial and terminal states are assigned as well as in the case when either is assigned and the other is weighted in the performance index. This method is based on a parametrization of all the state-costate functions satisfying the Hamiltonian differential equation.

The present paper is intended to be the counterpart of [5] for discrete-time systems. Though, the extension is not straightforward as we consider the general case when the matrix weighting the control input is possibly singular and without any assumptions on the invertibility of the state dynamics of the system. We refer to [3] and [12], for continuous and discrete-time singular LQ problems, respectively.

We remark that the problem considered in this paper is pretty general, since we allow each one of the initial and terminal states to be either fixed or quadratically weighted in the performance index, thus encompassing the standard LQ problem, in which the initial state is assigned and the terminal state is quadratically weighted, and the so-called fixed end-point LQ problem, in which the extreme states are both sharply assigned. We refer to [7], [14] and references therein for a discussion on the presence of constraints in the initial and terminal states. See also [2] for a feedback compensation scheme that guarantees optimality only for a given set of initial states. The contribution of this paper is that of providing closed-form expressions for the input and state evolutions, which depend only on the stabilizing solution of a discrete-time algebraic Riccati equation and on the solution of a symmetric Stein equation (also known as discretetime Lyapunov equation) and do not require the iteration of a difference Riccati equation. This result hinges on a parametrization of the solutions of the extended symplectic system associated with the optimal control problem. To obtain this parametrization, several ancillary results of independent interest on the discrete-time Riccati equation are derived.

The approach presented is particularly convenient for manifold reasons:

- Several versions of the LQ problem (encompassing the classical and the fixed end-point ones) are treated in a unified framework.

- The closed-form expression of the optimal trajectory and of the optimal cost can be exploited for the analysis of the structural properties of the optimal solutions and for solving more general (possibly parametric) optimization problems, e.g. problems having the LQ as a subproblem. For example, consider the case of two (or more) optimal control problems that are coupled by constraints or by a weight on the respective boundary conditions (see Section 5.1).

- The optimal state-costate trajectories and control law are expressed as functions of two stable matrices, thus ensuring the robustness of the obtained solution even for very large time-horizons.

- Standard software routines can be exploited for the solution of the algebraic Riccati and Stein equations.

Throughout this paper, the symbol $\mathbb{R}^{n \times m}$ denotes the space of $n \times m$ real matrices, $I$ denotes the identity matrix, $A^{+}$denotes the Moore-Penrose pseudoinverse of matrix $A$. The symbol $\sigma(A)$ denotes the spectrum of $A$. 


\section{Description of the problem}

Consider the linear time-invariant discrete-time state equation:

$$
x(k+1)=A x(k)+B u(k),
$$

where, for all $k \geq 0, x(k) \in \mathbb{R}^{n}$ is the state, $u(k) \in \mathbb{R}^{m}$ is the control input, $A \in \mathbb{R}^{n \times n}$ and $B \in \mathbb{R}^{n \times m}$. Consider an $n \times n$ matrix $Q$, an $m \times m$ matrix $R$ and an $n \times m$ matrix $S$ such that

$$
\Pi:=\left[\begin{array}{cc}
Q & S \\
S^{\top} & R
\end{array}\right]=\Pi^{\top} \geq 0 .
$$

The set of matrices $(A, B, \Pi)$ is often referred to as a Popov triplet, and will be denoted by $\Sigma$. Let $k_{f}>0$, and $P_{0}, P_{f} \in \mathbb{R}^{n \times n}$ be symmetric and positive semidefinite. Moreover, define the quadratic cost functional

$$
J(x, u):=x^{\top}(0) P_{0} x(0)+\sum_{k=0}^{k_{f}-1}\left[x^{\top}(k) u^{\top}(k)\right]\left[\begin{array}{cc}
Q & S \\
S^{\top} & R
\end{array}\right]\left[\begin{array}{l}
x(k) \\
u(k)
\end{array}\right]+x^{\top}\left(k_{f}\right) P_{f} x\left(k_{f}\right) .
$$

In this paper, we will consider the following optimal control problems.

Problem 1 (Assigned initial and terminal states). Given $x_{0}, x_{f} \in \mathbb{R}^{n}$, find a control law $u(k)$, $k \in\left\{0, \ldots, k_{f}-1\right\}$, minimizing (2) with $P_{0}=P_{f}=0$ under the constraints $x(0)=x_{0}, x\left(k_{f}\right)=x_{f}$ and (1).

Notice that in this case $k_{f}$ must be greater than the reachability index of the pair $(A, B)$ to guarantee the existence of the solution for any $x_{0}, x_{f} \in \mathbb{R}^{n}$.

Problem 2 (Assigned initial state and weighted terminal state). Given $x_{0} \in \mathbb{R}^{n}$, find $u(k)$, $k \in\left\{0, \ldots, k_{f}-1\right\}$, minimizing (2) with $P_{0}=0$ under the constraints $x(0)=x_{0}$ and (1).

Problem 3 (Assigned terminal state and weighted initial state). Given $x_{f} \in \mathbb{R}^{n}$, find $u(k)$, $k \in\left\{0, \ldots, k_{f}-1\right\}$, minimizing (2) with $P_{f}=0$ under the constraints $x\left(k_{f}\right)=x_{f}$ and (1).

Problem 4 (Weighted initial and terminal states). Find $u(k), k \in\left\{0, \ldots, k_{f}-1\right\}$, minimizing (2) under the constraint (1).

In the case of Problem 4, a trivial optimal solution is the identically zero state trajectory and control law.

\section{Mathematical background and preliminary results}

It is well-known (see e.g. [6], [9]) that the optimal state trajectory and control law satisfy the extended symplectic system $\operatorname{ESS}(\Sigma)$

$$
\begin{aligned}
& x(k+1)=A x(k)+B u(k), \\
& \lambda(k)=Q x(k)+A^{\top} \lambda(k+1)+S u(k), \\
& 0=S^{\top} x(k)+B^{\top} \lambda(k+1)+R u(k),
\end{aligned}
$$

with $k \in\left\{0, \ldots, k_{f}-1\right\}$, which is obtained by extending the state $x(k)$ of system (1) with the costate $\lambda(k) \in \mathbb{R}^{n}$. More precisely, if $x(k)$ is the optimal state trajectory of any of the Problems 1-4 (whatever the assigned initial or terminal conditions), then a costate trajectory $\lambda(k)$ and an 
input function $u(k)$ exist such that the vector $\left[x^{\top}(k) \lambda^{\top}(k) u^{\top}(k)\right]^{\top}$ satisfies (3)-(5). Notice that (3)-(5) can be written in the descriptor form

$$
F p(k+1)=G p(k), \quad k \in\left\{0, \ldots, k_{f}-1\right\},
$$

where

$$
F:=\left[\begin{array}{ccc}
I & 0 & 0 \\
0 & -A^{\top} & 0 \\
0 & -B^{\top} & 0
\end{array}\right], \quad G:=\left[\begin{array}{ccc}
A & 0 & B \\
Q & -I & S \\
S^{\top} & 0 & R
\end{array}\right] \quad \text { and } \quad p(k):=\left[\begin{array}{c}
x(k) \\
\lambda(k) \\
u(k)
\end{array}\right]
$$

The matrix pencil $z F-G$ is known as the extended symplectic pencil associated with $\Sigma$.

Remark 1 If the extended symplectic pencil $z F-G$ is regular, the system of equations (6) has $2 n+m$ linearly independent solutions, spanning a $(2 n+m)$-dimensional space (see e.g. [4] and [10]). Notice however that, since $u\left(k_{f}\right)$ is irrelevant both to the value of the cost function and to the satisfaction of the constraints (for each one of the four optimal control problems considered), we may regard as equivalent two solutions $p_{1}(k)$ and $p_{2}(k)$ of $(6)$ if they only differ for the value of $u\left(k_{f}\right)$. On the other hand, a solution $p(k)$ of $(6)$ remains such when we exchange $u\left(k_{f}\right)$ with an arbitrary $\bar{u} \in \mathbb{R}^{m}$. Therefore, the dimension of the space of non-equivalent solutions of (6) equals $2 n$. We set as a representative element of each equivalence class of the solutions of (6) the vector $p(k)$ such that the corresponding $u\left(k_{f}\right)$ is zero.

We make the following standing assumptions.

(A1) the pair $(A, B)$ is reachable;

(A2) The extended symplectic pencil $z F-G$ associated to $\Sigma$ is regular and has no generalized eigenvalues on the unit circle.

Moreover, without loss of generality we may assume that $\Theta:=\left[\begin{array}{ll}B^{\top} & R\end{array}\right]^{\top}$ is full-column rank. Indeed, if $\Theta$ has non-trivial kernel, there exists a subspace $\mathcal{U}_{0}$ of the input space that does not influence the state dynamics and the cost function. Then, by performing a suitable (orthogonal) change of basis in the input space, we may eliminate $\mathcal{U}_{0}$ and obtain an equivalent problem for which this condition is satisfied.

Recall that the discrete-time algebraic Riccati equation DARE $(\Sigma)$

$$
P=A^{\top} P A-\left(A^{\top} P B+S\right)\left(R+B^{\top} P B\right)^{-1}\left(B^{\top} P A+S^{\top}\right)+Q
$$

has a (unique) symmetric positive semidefinite solution $P_{+}$such that all the eigenvalues of the closed-loop matrix

$$
A_{+}=A-B\left(R+B^{\top} P_{+} B\right)^{-1}\left(B^{\top} P_{+} A+S^{\top}\right)
$$

lie inside the unit disk if and only if the pair $(A, B)$ is stabilizable and assumption (A2) holds, and a (unique) symmetric negative semidefinite solution $P_{-}$such that all the eigenvalues of

$$
A_{-}=A-B\left(R+B^{\top} P_{-} B\right)^{-1}\left(B^{\top} P_{-} A+S^{\top}\right)
$$

are outside the unit disk if assumptions (A1)-(A2) hold and in addition the extended symplectic pencil $z F-G$ has no generalized eigenvalues at zero.

The matrices $P_{+}$and $P_{-}$, when they exist, are respectively called the stabilizing and antistabilizing solutions of the DARE $(\Sigma)$. 
If $A$ is invertible, we define as in [6, p.105] the time-reversed system associated with (1) as

$$
x(k)=\bar{A} x(k+1)+\bar{B} u(k),
$$

where $\bar{A}=A^{-1}$ and $\bar{B}=-A^{-1} B$. The weighting matrix associated with (10) is

$$
\bar{\Pi}:=\left[\begin{array}{cc}
\bar{Q} & \bar{S} \\
\bar{S}^{\top} & \bar{R}
\end{array}\right]
$$

where $\bar{Q}:=A^{-\top} Q A^{-1}, \bar{R}:=R-S^{\top} A^{-1} B-B^{\top} A^{-\top} S+B^{\top} A^{-\top} Q A^{-1} B$ and $\bar{S}:=A^{-\top} S-$ $A^{-\top} Q A^{-1} B$. The set of matrices $\bar{\Sigma}:=(\bar{A}, \bar{B}, \bar{\Pi})$ is the time-reversed Popov triplet. The link between the extreme solutions of the $\operatorname{DARE}(\Sigma)$ and $\operatorname{DARE}(\bar{\Sigma})$ is described by the following theorem (see e.g. [6, p.106]).

Theorem 1 Let $A$ be invertible. Let $\bar{P}$ be a symmetric solution of the DARE $(\bar{\Sigma})$. Then $-\bar{P}$ is a solution of the DARE $(\Sigma)$ if and only if the extended symplectic pencil $z F-G$ is regular and has no generalized eigenvalues at zero or, equivalently, if and only if $R-S^{\top} A^{-1} B$ is non-singular.

Recall the following theorem about the Stein equation.

Theorem 2 Let $A \in \mathbb{R}^{n \times n}$ and $B \in \mathbb{R}^{n \times m}$. The symmetric Stein equation

$$
A X A^{\top}-X+B B^{\top}=0
$$

has a unique symmetric solution $X \in \mathbb{R}^{n \times n}$ if and only if the spectrum of $A$ does not contain reciprocal pairs, i.e., if and only if $\lambda \in \sigma(A)$ implies that $\lambda^{-1} \notin \sigma(A)$. Moreover, if the pair $(A, B)$ is reachable, then $A$ is a stability matrix if and only if (11) admits a unique positive definite solution.

In the following theorem, we present an efficient method for the computation of the maximal solution $\bar{P}_{+}$of the time-reversed DARE and of the corresponding closed-loop system matrix $\bar{A}_{+}$, which is based on the solution of a symmetric Stein equation. This result is an extension of a well-known property of continuous-time systems (see [13, p.354]). Then, in Section 4, it will be shown that, even when the system matrix $A$ is singular and the time-reversed system $\bar{\Sigma}$ is not defined, these formulas still provide two matrices, say $P_{*}$ and $A_{*}$, that can be exploited to derive a parametrization of all the solutions of the extended symplectic system.

Theorem 3 Let $A$ be invertible. Let $P_{+}$and $\bar{P}_{+}$be the positive semidefinite solutions of the $D A R E(\Sigma)$ and DARE $(\bar{\Sigma})$, respectively. Then, the matrix $\Delta:=P_{+}+\bar{P}_{+}$is positive definite and it is the inverse of the unique solution $W$ of the Stein equation

$$
A_{+} W A_{+}^{\top}-W+B\left(R+B^{\top} P_{+} B\right)^{-1} B^{\top}=0 .
$$

Proof: First, suppose that $L:=R-S^{\top} A^{-1} B$ is invertible. In this case the $\operatorname{DARE}(\bar{\Sigma})$ has a maximal positive semidefinite solution $\bar{P}_{+}$. By virtue of Theorem 1 , the matrix $-\bar{P}_{+}$is a solution of the DARE $(\Sigma)$, and being negative semidefinite it is indeed $P_{-}$. Hence, $\Delta$ equals the difference $P_{+}-P_{-}$, and satisfies the homogeneous algebraic Riccati equation

$$
\Delta=A_{+}^{\top} \Delta A_{+}+A_{+}^{\top} \Delta B R_{1}^{-1} B^{\top} \Delta A_{+},
$$

where $R_{1}:=R+B^{\top} P_{+} B-B^{\top} \Delta B$; moreover, $\Delta_{0}$ is a solution of (13) if and only if $\Delta_{0}=P_{+}-P_{0}$, where $P_{0}$ is a solution of the $\operatorname{DARE}(\Sigma)$ (see [16, Lemma 3.2]). Now, consider the Stein equation (12). Since $(A, B)$ is reachable, $\left(A_{+}, B\right)$ is such, and hence $\left(A_{+}, B\left(R+B^{T} P_{+} B\right)^{-\frac{1}{2}}\right)$ remains 
reachable in view of the non-singularity of $\left(R+B^{T} P_{+} B\right)^{-\frac{1}{2}}$. By virtue of Theorem 2 , it follows that equation (12) does admit a unique solution $W$, and this solution is positive definite. Moreover, since the extended symplectic pencil is regular and has no generalized eigenvalues at zero, we may conclude that $A_{+}$is non-singular. It follows that (12) can be written as

$$
\begin{aligned}
W & =A_{+}^{-1} W A_{+}^{-\top}-A_{+}^{-1} B\left(R+B^{\top} P_{+} B-B^{\top} W^{-1} B+B^{\top} W^{-1} B\right)^{-1} B^{\top} A_{+}^{-\top} \\
& =A_{+}^{-1} W A_{+}^{-\top}-A_{+}^{-1} \Xi B\left(R+B^{\top} P_{+} B-B^{\top} W^{-1} B+B^{\top} W^{-1} A_{+} A_{+}^{-1} \Xi B\right)^{-1} B^{\top} \Xi^{\top} A_{+}^{-\top},
\end{aligned}
$$

where $\Xi:=W A_{+}^{-\top} A_{+}^{\top} W^{-1}=I$. Both sides of the former can be inverted, and by applying the well-known matrix inversion lemma, we get

$$
W^{-1}=A_{+}^{\top} W^{-1} A_{+}+A_{+}^{\top} W^{-1} B R_{1}^{-1} B^{\top} W^{-1} A_{+} .
$$

Conversely, the same calculation in the reverse order shows that if $\bar{\Delta}$ is a non-singular solution of (13), then $\bar{\Delta}^{-1}$ is a solution of (12). As a result, $W^{-1}$ is the unique positive definite solution of (13). Now we show that $W^{-1}=\Delta$. As already observed, since $W^{-1}$ is a solution of (13), we have $W^{-1}=P_{+}-P_{0}$, where $P_{0}$ is a solution of the $\operatorname{DARE}(\Sigma)$. On the other hand, $P_{-}$is the minimal solution of the $\operatorname{DARE}(\Sigma)$, hence $W^{-1}=P_{+}-P_{0} \leq P_{+}-P_{-}=\Delta$. It follows that $\Delta$ is positive definite as well. Since $W^{-1}$ is the unique positive definite solution of (13), it follows that $\Delta=W^{-1}$.

Now, let $L$ be singular. A real $\delta>0$ can be found such that, for any $\varepsilon \in(0, \delta)$, by defining $R_{\varepsilon}:=R+\varepsilon I_{m}$, the matrix

$$
L_{\varepsilon}:=R_{\varepsilon}-S^{\top} A^{-1} B
$$

is non-singular. Choose for example $\delta:=\min \{|\mathfrak{R e}\{\lambda\}| \mid \lambda \in \sigma(L) \backslash\{0\}\}$. Note that for all $\varepsilon \in[0, \delta)$ the matrix

$$
\Pi_{\varepsilon}:=\left[\begin{array}{cc}
Q & S \\
S^{\top} & R_{\varepsilon}
\end{array}\right]
$$

is symmetric positive semidefinite. In fact, more is true: for all $0 \leq \varepsilon_{1} \leq \varepsilon_{2}<\delta$ we have $0 \leq \Pi \leq \Pi_{\varepsilon_{1}} \leq \Pi_{\varepsilon_{2}}$. Let $\Sigma_{\varepsilon}:=\left(A, B, \Pi_{\varepsilon}\right)$ and $\bar{\Sigma}_{\varepsilon}$ be the corresponding time-reversed Popov triplet. Since $\Pi_{\varepsilon}=\Pi_{\varepsilon}^{\top} \geq 0$, two matrices $C_{\varepsilon}$ and $D_{\varepsilon}$ exist such that $\Pi_{\varepsilon}$ can be partitioned as $\Pi_{\varepsilon}=F_{\varepsilon}^{\top} F_{\varepsilon}$, with $F_{\varepsilon}:=\left[\begin{array}{cc}C_{\varepsilon} & D_{\varepsilon}\end{array}\right]$, where $D_{\varepsilon}$ is full-column rank since $R_{\varepsilon}=D_{\varepsilon}^{\top} D_{\varepsilon}>0$ for all $\varepsilon$ in $(0, \delta)$. The injectivity of $D_{\varepsilon}$ for all $\varepsilon \in(0, \delta)$, assumptions (A1)-(A2) and the non-singularity of $L_{\varepsilon}$ ensure the existence and uniqueness of the positive semidefinite solution of the DARE $\left(\Sigma_{\varepsilon}\right)$ and of the $\operatorname{DARE}\left(\bar{\Sigma}_{\varepsilon}\right)$, here denoted by $P_{+, \varepsilon}$ and $\bar{P}_{+, \varepsilon}$, respectively. Moreover, $P_{+, \varepsilon}$ and $\bar{P}_{+, \varepsilon}$ are continuous in $[0, \delta)$ since the mapping $\varepsilon \longrightarrow \Pi_{\varepsilon}$ is continuous in $[0, \delta)$ and monotonically non-decreasing on $[0, \delta)$, i.e. $P_{+, \varepsilon} \longrightarrow P_{+, 0}=P_{+}$and $\bar{P}_{+, \varepsilon} \longrightarrow \bar{P}_{+, 0}=\bar{P}_{+}$as $\varepsilon \longrightarrow 0^{+}\left(\operatorname{see}^{1}\left[17\right.\right.$, p.107]). Let $A_{+, \varepsilon}$ be the stable closed-loop matrix corresponding to $P_{+, \varepsilon}$ and let $V_{\varepsilon}:=B\left(R_{\varepsilon}+B^{\top} P_{+} B\right)^{-1} B^{\top}$. Note that $A_{+, \varepsilon}$ and $V_{\varepsilon}$ are continuous in $[0, \delta)$ as $R_{\varepsilon}$ and $P_{+, \varepsilon}$ are continuous in $[0, \delta)$. It follows that the solution $W_{\varepsilon}$ of the Stein equation

$$
A_{+, \varepsilon} W_{\varepsilon} A_{+, \varepsilon}^{\top}-W_{\varepsilon}+V_{\varepsilon}=0
$$

is continuous in $[0, \delta)$. Moreover, $W_{\varepsilon}$ is invertible by virtue of Theorem 2 since the pair $\left(A_{+, \varepsilon}, V_{\varepsilon}\right)$ is reachable, as can be proved by using the same arguments presented in the case of $L$ invertible. It follows that $W_{\varepsilon}$ is invertible by virtue of Theorem 2 , and its inverse $\Delta_{\varepsilon}:=W_{\varepsilon}^{-1}$ is continuous in $[0, \delta)$. Now, since for all $\varepsilon \in(0, \delta)$ the matrix $L_{\varepsilon}$ is non-singular, it follows that $P_{+, \varepsilon}+\bar{P}_{+, \varepsilon}=\Delta_{\varepsilon}$

\footnotetext{
${ }^{1}$ In $\left[17\right.$, p.107], the further assumption of full-column rankness of matrix $D_{0}$ is not necessary here: in fact in that context the injectivity of matrix $D_{\varepsilon}$ for all $\varepsilon \in[0, \delta)$ guarantees the existence and uniqueness of the positive semidefinite solution of the $\operatorname{DARE}\left(\Sigma_{\varepsilon}\right)$, which is here ensured by assumptions (A1)-(A2).
} 
for all $\varepsilon \in(0, \delta)$. Therefore, since the two functions $P_{+, \varepsilon}+\bar{P}_{+, \varepsilon}$ and $\Delta_{\varepsilon}$ are continuous in $[0, \delta)$ and they are equal in $(0, \delta)$, then they are equal at $\varepsilon=0$ as well. As a consequence, $\Delta_{0}=P_{+, 0}+\bar{P}_{+, 0}=P_{+}+\bar{P}_{+}=\Delta$. By the continuity of $\Delta_{\varepsilon}$ in $[0, \delta)$ it follows that $\Delta^{-1}$ is the unique solution of (15) at $\varepsilon=0$, which is exactly (12).

Theorem 4 Let $A$ be invertible. Let $P_{+}$and $\bar{P}_{+}$be the positive semidefinite solutions of the $D A R E(\Sigma)$ and DARE $(\bar{\Sigma})$, respectively. Let $\bar{A}_{+}$be the stable closed-loop matrix of the timereversed system, defined by

$$
\bar{A}_{+}:=\bar{A}-\bar{B}\left(\bar{R}+\bar{B}^{\top} \bar{P}_{+} \bar{B}\right)^{-1}\left(\bar{B}^{\top} \bar{P}_{+} \bar{A}+\bar{S}^{\top}\right) .
$$

The following relation holds:

$$
\bar{A}_{+}=\Delta^{-1} A_{+}^{\top} \Delta .
$$

Proof: First suppose that $L:=R-S^{\top} A^{-1} B$ is invertible. As already seen in the proof of Theorem 3 , in this case we have $P_{-}=-\bar{P}_{+}$. Define (as it is done in [6, p.107])

$$
G\left(P_{-}\right):=R+B^{\top} P_{-} B, \quad H\left(P_{-}\right):=A^{\top} P_{-} B+S, \quad K\left(P_{-}\right):=A^{\top} P_{-} A-P_{-}+Q .
$$

By exploiting the DARE $(\Sigma)$, that can be written as $K(P)=H(P) G^{-1}(P) H^{\top}(P)$, and the identity $L=G\left(P_{-}\right)-H^{\top}\left(P_{-}\right) A^{-1} B$ (see $[6$, p.107]), we get

$$
\begin{aligned}
L^{\top} G^{-1}\left(P_{-}\right) H^{\top}\left(P_{-}\right) & =\left(G\left(P_{-}\right)-H^{\top}\left(P_{-}\right) A^{-1} B\right)^{\top} G^{-1}\left(P_{-}\right) H^{\top}\left(P_{-}\right)= \\
& =H^{\top}\left(P_{-}\right)-B^{\top} A^{-\top} K\left(P_{-}\right)= \\
& =S^{\top}+B^{\top} A^{-\top} P_{-}-B^{\top} A^{-\top} Q .
\end{aligned}
$$

yielding the following identity:

$$
\left(R-B^{\top} A^{-\top} S\right)^{-1}\left(S^{\top}+B^{\top} A^{-\top} P_{-}-B^{\top} A^{-\top} Q\right)=\left(R+B^{\top} P_{-} B\right)^{-1}\left(B^{\top} P_{-} A+S^{\top}\right) .
$$

On the other hand, notice that $\bar{A}_{+}^{-1}$, in view of the well-known matrix inversion lemma, can be expressed as

$$
\begin{aligned}
\bar{A}_{+}^{-1} & =A+A \bar{B}\left(\bar{R}-\bar{B}^{\top} P_{-} \bar{B}-\left(\bar{S}^{\top}-\bar{B}^{\top} P_{-} \bar{A}\right) A \bar{B}\right)^{-1}\left(\bar{S}^{\top}-\bar{B}^{\top} P_{-} \bar{A}\right) A= \\
& =A-B\left(\bar{R}+\bar{S}^{\top} B\right)^{-1}\left(\bar{S}^{\top} A-\bar{B}^{\top} P_{-}\right)= \\
& =A-B\left(R-B^{\top} A^{-\top} S\right)^{-1}\left(S^{\top}+B^{\top} A^{-\top} P_{-}-B^{\top} A^{-\top} Q\right) .
\end{aligned}
$$

Hence, by taking into account the latter, (9) and (18), it follows that $A_{-}=\bar{A}_{+}^{-1}$. On the other hand, by using (8) and (9) and by exploiting the fact that both $P_{+}$and $P_{-}$are solutions of the $\operatorname{DARE}(\Sigma)$, it can be checked that $A_{-}=\Delta^{-1} A_{+}^{-\top} \Delta$. Hence (17) holds.

Let now $L$ be singular. Consider a real $\delta>0$ such that for any $\varepsilon \in(0, \delta)$ the matrix $L_{\varepsilon}$ defined in (14) is non-singular. Let $\Sigma_{\varepsilon}$ be defined as in the proof of Theorem 3 and let $\bar{\Sigma}_{\varepsilon}$ be its timereversed representation. The existence and uniqueness of the positive semidefinite solutions of the $\operatorname{DARE}\left(\Sigma_{\varepsilon}\right)$ and $\operatorname{DARE}\left(\bar{\Sigma}_{\varepsilon}\right)$, say $P_{+, \varepsilon}$ and $\bar{P}_{+, \varepsilon}$, respectively, and their continuity in $[0, \delta)$ was proved in the proof of Theorem 3. It was also shown that their difference $\Delta_{\varepsilon}$ is continuous and positive definite in $[0, \delta)$, whereas $A_{+, \varepsilon}$ is continuous in $[0, \delta)$. As a consequence, the product $\Delta_{\varepsilon}^{-1} A_{+, \varepsilon}^{\top} \Delta_{\varepsilon}$ is continuous in $[0, \delta)$. Moreover, since $\bar{A}_{+, \varepsilon}$ is defined by applying (16) to the time-reversed representation of $\Sigma_{\varepsilon}, \bar{A}_{+, \varepsilon}$ is obviously continuous in $[0, \delta)$. The functions $\bar{A}_{+, \varepsilon}$ and $\Delta_{\varepsilon}^{-1} A_{+, \varepsilon}^{\top} \Delta_{\varepsilon}$ are continuous in $[0, \delta)$ and equal in $(0, \delta)$. It follows that they are equal at $\varepsilon=0$. Hence (17) follows. 


\section{Optimal state-costate trajectories and control law}

In this section, it is first shown how the solutions of the extended symplectic system can be parametrized in closed-form. Then, as a second step, we show how to exploit such parametrization for the solution of Problems 1-4.

\subsection{Solutions of the extended symplectic system}

The following theorem parametrizes the set of representatives (in the sense of Remark 1) of the solutions of the extended symplectic system (6) in terms of two $n$-dimensional vectors $p$ and $q$.

Theorem 5 Let assumptions (A1)-(A2) hold. Denote by $P_{+}$the stabilizing solution of the $D A R E(\Sigma)$ and by $\Delta$ the inverse of the (unique) positive definite solution of the Stein equation (12). Let $P_{*}:=\Delta-P_{+}$,

$$
A_{*}:=\Delta^{-1} A_{+}^{\top} \Delta
$$

and let $K_{+}$and $K_{*}$ be the stabilizing gains defined by

$$
\begin{aligned}
K_{+} & :=\left(R+B^{\top} P_{+} B\right)^{-1}\left(B^{\top} P_{+} A+S^{\top}\right), \\
K_{*} & :=\left(R+B^{\top} B\right)^{-1}\left(B^{\top}\left(A A_{*}-I\right)+S^{\top} A_{*}-B^{\top} P_{*}\right) .
\end{aligned}
$$

The set of representatives of the solutions of the extended symplectic system (6) is parametrized by the following expression in terms of $p, q \in \mathbb{R}^{n}$ :

$$
\left[\begin{array}{c}
x(k) \\
\lambda(k) \\
u(k)
\end{array}\right]= \begin{cases}{\left[\begin{array}{c}
I \\
P_{+} \\
-K_{+}
\end{array}\right] A_{+}^{k} p+\left[\begin{array}{c}
A_{*} \\
-P_{*} A_{*} \\
-K_{*}
\end{array}\right] A_{*}^{k_{f}-k-1} q} & 0 \leq k \leq k_{f}-1 \\
{\left[\begin{array}{c}
I \\
P_{+} \\
0
\end{array}\right] A_{+}^{k_{f}} p+\left[\begin{array}{c}
I \\
-P_{*} \\
0
\end{array}\right] q} & k=k_{f}\end{cases}
$$

Proof: First, suppose $A$ to be invertible. By virtue of Theorems 3 and 4 it is found that $P_{*}=\bar{P}_{+}$and $A_{*}=\bar{A}_{+}$. Now we prove that $K_{*}=\bar{K}_{+}$, where $\bar{K}_{+}$is the stabilizing gain matrix for $\bar{\Sigma}$, defined as

$$
\bar{K}_{+}:=\left(\bar{R}+\bar{B}^{\top} \bar{P}_{+} \bar{B}\right)^{-1}\left(\bar{B}^{\top} \bar{P}_{+} \bar{A}+\bar{S}^{\top}\right) .
$$

As a consequence of (23) and of $A_{*}=\bar{A}_{+}$we have

$$
A A_{*}-B \bar{K}_{+}=I,
$$

that can be proved by direct substitution. The DARE $(\bar{\Sigma})$ can be written as

$$
A^{-\top}\left(P_{*}+Q\right) A^{-1}-P_{*}-\left(A^{-\top} S-A^{-\top}\left(P_{*}+Q\right) A^{-1} B\right) \bar{K}_{+}=0 .
$$

By premultiplying (25) by $B^{\top}$ and by adding the identity thus obtained with (23), one gets

$$
B^{\top} P_{*}=S^{\top} A_{*}-R \bar{K}_{+} .
$$

From the injectivity of $\left[B^{\top} R\right]^{\top}$, it follows that $B^{\top} B+R$ is invertible. Hence, by premultiplying (24) by $B^{\top}$ and by taking into account (26), we get $\bar{K}_{+}=K_{*}$. 
We are now ready to show that $(22)$ are indeed solutions of the $\operatorname{ESS}(\Sigma)$. First, let $k<k_{f}-1$. Substitute (22) in (6), and notice that the terms in $p$ are equal since

$$
\left[\begin{array}{c}
I \\
-A^{\top} P_{+} \\
-B^{\top} P_{+}
\end{array}\right] A_{+}=\left[\begin{array}{c}
A-B K_{+} \\
Q-P_{+}-S K_{+} \\
S^{\top}-R K_{+}
\end{array}\right]
$$

Indeed, the first block-row equation holds by definition of $A_{+}$and the remaining are equivalent to (7) with (20). Likewise, the terms in $q$ are equal since

$$
\left[\begin{array}{c}
I \\
A^{\top} P_{*} \\
B^{\top} P_{*}
\end{array}\right] A_{*}=\left[\begin{array}{c}
A A_{*}-B K_{*} \\
Q A_{*}+P_{*} A_{*}-S K_{*} \\
S^{\top} A_{*}-R K_{*}
\end{array}\right] A_{*}
$$

holds owing to (24), (26) and by noticing that the second block-row equality follows by premultiplying (25) by $-A^{\top}$.

Let now $k=k_{f}-1$. Since (27) and (28) still hold, relation (22) satisfies (6) for $k=k_{f}-1$ as well.

Now, let $A$ be singular. A preliminary state feedback $u(k)=F x(k)$ can be performed so as to obtain a non-singular system matrix $A_{F}:=A+B F$ (note that this is always possible since $(A, B)$ is reachable). As shown in [6, p.96], to this feedback transformation there corresponds a new Popov triplet $\Sigma_{F}:=\left(A_{F}, B_{F}, \Pi_{F}\right)$ with $B_{F}=B$ and

$$
\Pi_{F}:=\left[\begin{array}{cc}
Q_{F} & S_{F} \\
S_{F}^{\top} & R_{F}
\end{array}\right]
$$

where $Q_{F}:=Q+S F+F^{\top} S^{\top}+F^{\top} R F, R_{F}:=R$ and $S_{F}:=S+F^{\top} R$. Notice that $P_{+}$is the maximal solution of the $\operatorname{DARE}(\Sigma)$ if and only if it is the maximal solution of the $\operatorname{DARE}\left(\Sigma_{F}\right)$ for all $F$ (see [6, p.100]). Moreover, $A_{+}, \Delta$ and $A_{*}$ are independent of $F$. As a consequence, equation (19) provides the stable closed-loop system matrix of the time-reversed representation of $\Sigma_{F}$, say $\bar{\Sigma}_{F}$, for any $F$ such that $A_{F}$ is non-singular. Hence, equation (22), written with respect to $\Sigma_{F}$ and to $\bar{\Sigma}_{F}$, furnishes for all $p, q \in \mathbb{R}^{n}$ solutions of the $\operatorname{ESS}\left(\Sigma_{F}\right)$. However, by taking into account (3)-(5), it can be easily checked that $p_{F}(k):=\left[\begin{array}{lll}x_{F}^{\top}(k) & \lambda_{F}^{\top}(k) & u_{F}^{\top}(k)\end{array}\right]^{\top}$ is a solution of the $\operatorname{ESS}\left(\Sigma_{F}\right)$, if and only if $p(k):=\left[\begin{array}{lll}x^{\top}(k) & \lambda^{\top}(k) & u^{\top}(k)\end{array}\right]^{\top}$, where $x(k):=x_{F}(k), \lambda(k):=\lambda_{F}(k)$ and $u(k):=u_{F}(k)+F x_{F}(k)$, is a solution of the $\operatorname{ESS}(\Sigma)$. Notice that

$$
\left[\begin{array}{c}
I \\
-A_{F}^{\top} P_{+} \\
-B_{F}^{\top} P_{+}
\end{array}\right] A_{+}=\left[\begin{array}{c}
A_{F}-B_{F}\left(K_{+}+F\right) \\
Q_{F}-P_{+}-S_{F}\left(K_{+}+F\right) \\
S_{F}^{\top}-R\left(K_{+}+F\right)
\end{array}\right],
$$

which follows after simple algebraic manipulations: the equality of the first block-row derives from the definition of $A_{+}$; the second is implied by the relation obtained by (27) written with respect to $\Sigma_{F}$ and by the definition of $K_{+}$; the third follows from (27) as well. Moreover, there holds

$$
\left[\begin{array}{c}
I \\
A_{F}^{\top} P_{*} \\
B_{F}^{\top} P_{*}
\end{array}\right] A_{*}=\left[\begin{array}{c}
A_{F} A_{*}-B_{F}\left(K_{*}+F A_{*}\right) \\
Q_{F} A_{*}+P_{*} A_{*}-S_{F}\left(K_{*}+F A_{*}\right) \\
S_{F}^{\top} A_{*}-R_{F}\left(K_{*}+F\right)
\end{array}\right] A_{*}
$$

that follows from (28) written with respect to $\Sigma_{F}$. As a result of (29) and (30), it follows that for all $k \in\left\{0, \ldots, k_{f}-1\right\}$

$$
\left[\begin{array}{c}
x_{F}(k) \\
\lambda_{F}(k) \\
u_{F}(k)
\end{array}\right]=\left[\begin{array}{c}
I \\
P_{+} \\
-K_{+}-F
\end{array}\right] A_{+}^{k} p+\left[\begin{array}{c}
A_{*} \\
-P_{*} A_{*} \\
-K_{*}-F A_{*}
\end{array}\right] A_{*}^{k_{f}-k-1} q
$$


for any $p$ and $q$ in $\mathbb{R}^{n}$. Equation (31) shows that the vector $p(k)$ defined above is a trajectory of (22).

Conversely, let us show that all functions satisfying the $\operatorname{ESS}(\Sigma)$ can be expressed by means of (22). To this aim, we show that (22) has $2 n$ linearly independent trajectories. Let in fact

$$
V_{1}:=\left[\begin{array}{c}
I \\
P_{+} \\
-K_{+}
\end{array}\right], \quad V_{2}:=\left[\begin{array}{c}
A_{*} \\
-P_{*} A_{*} \\
-K_{*}
\end{array}\right] .
$$

It is easy to verify that $F V_{1} A_{+}=G V_{1}$ and $F V_{2}=G V_{2} A_{*}$, where $F$ and $G$ are defined in Section 3. It follows that $V_{1}$ and $V_{2}$ are basis matrices for two deflating subspaces of the symplectic pencil $z F-G$, since $A_{+}-\lambda I$ and $I-\lambda A_{*}$ are both regular pencils (see e.g. [15]). From [9, Theorem 1.6.5], it follows that $\mathcal{V}:=\operatorname{im} V_{1} \cap \operatorname{im} V_{2}$ is still a deflating subspace of $z F-G$. Moreover, since the generalized eigenvalues of $z F-G$ corresponding to the deflating subspaces im $V_{1}$ and $\operatorname{im} V_{2}$ are the eigenvalues of $A_{+}$(hence stable) and the reciprocal of the eigenvalues of $A_{*}$ (hence with modulus greater than 1 and possibly infinity), respectively, the set of generalized eigenvalues of $z F-G$ corresponding to $\mathcal{V}$ is empty. Hence, $\mathcal{V}=\{0\}$. Hence, for any given pair $p, q \in \mathbb{R}^{n}$, the two trajectories $V_{1} A_{+}^{k} p$ and $V_{2} A_{*}^{k_{f}-k-1} q$ are linearly independent. Therefore, the dimension of the linear space of trajectories in (22) is given by the sum of the dimensions $n_{1}$ and $n_{2}$ of the subspaces of trajectories of (22) corresponding to $p=0$ and to $q=0$, respectively. Setting $k=0$ and $k=k_{f}$, we easily see that $n_{1}=n$ and $n_{2}=n$, respectively. In conclusion, (22) has $2 n$ linearly independent solutions. Since the dimension of the linear space of non-equivalent solutions of the $\operatorname{ESS}(\Sigma)$ is exactly $2 n$ (see Remark 1), it follows that (22) represents the complete set of solutions of the $\operatorname{ESS}(\Sigma)$ with $u\left(k_{f}\right)=0$.

\subsection{Optimal trajectories}

In Theorem 5 it has been shown that under assumptions (A1)-(A2) the matrices $P_{+}$and $P_{*}$ yield an explicit formula parametrizing in terms of $p$ and $q$ all the solutions of the extended symplectic system (6), which is a set of necessary conditions for an optimum. Now, from this set of trajectories we select those that satisfy the boundary conditions, so that the trajectories thus obtained are solutions of the complete set of Pontryagin equations written for the optimal control problem considered. Notice that, in general, the Pontryagin equations are only necessary for an optimum. However, in the present case they are also sufficient, by the convexity of Problems 1-4 and by the reachability of $(A, B)$ (the latter ensuring that a state and input functions satisfying the constraints of any of Problems 1-4 exist).

Now we show how to compute the values of the parameters $p$ and $q$ in terms of the boundary conditions. Let $\pi:=\left[\begin{array}{ll}p^{\top} & q^{\top}\end{array}\right]^{\top}$.

Problem 1 (Assigned initial and final states).

In this case the boundary equations are $x(0)=x_{0}$ and $x\left(k_{f}\right)=x_{f}$. By evaluating $(22)$ at $k=0$ and $k=k_{f}$ we get

$$
\xi_{1}=N_{1} \pi \quad \text { where } \quad \xi_{1}:=\left[\begin{array}{c}
x_{0} \\
x_{f}
\end{array}\right] \quad \text { and } \quad N_{1}:=\left[\begin{array}{cc}
I & A_{*}^{k_{f}} \\
A_{+}^{k_{f}} & I
\end{array}\right] .
$$

The corresponding values of $p$ and $q$ can be computed by matrix inversion, since $N_{1}$ is invertible. In fact, for any $x_{0}, x_{f} \in \mathbb{R}^{n}$, a state trajectory such that $x(0)=x_{0}$ and $x\left(k_{f}\right)=x_{f}$ exists since $k_{f}$ is supposed to be greater than the reachability index. Hence, an optimal trajectory for Problem 1 exists by the convexity of Problem 1 and, as a result, there exist $p$ and $q$ such that (32) holds. The arbitrariness of $x_{0}$ and $x_{f}$ implies that $N_{1}$ is invertible. 
Problem 2 (Assigned initial state and weighted terminal state).

Since the terminal state is quadratically weighted in the performance index, the Pontryagin set of equations includes a boundary condition on $\lambda\left(k_{f}\right)$, expressed by $\lambda\left(k_{f}\right)=P_{f} x\left(k_{f}\right)$. From this equation and from (22) we obtain

$$
\left(P_{f}-P_{+}\right) A_{+}^{k_{f}} p+\left(P_{f}+P_{*}\right) q=0 .
$$

Equation $x(0)=x_{0}$ and (33), in view of $(22)$ evaluated at $k=0$, leads to the compact expression

$$
\xi_{2}=N_{2} \pi \quad \text { where } \quad \xi_{2}:=\left[\begin{array}{c}
x_{0} \\
0
\end{array}\right] \quad \text { and } \quad N_{2}:=\left[\begin{array}{cc}
I & A_{*}^{k_{f}} \\
\left(P_{f}-P_{+}\right) A_{+}^{k_{f}} & P_{f}+P_{*}
\end{array}\right] .
$$

If $N_{2}$ is invertible, $p$ and $q$ are obtained from the former by inversion. If $N_{2}$ is singular, the convexity of Problem 2 ensures the existence of an optimal state trajectory. Hence $\xi_{2} \in \operatorname{im} N_{2}$. As a consequence, the set of all parameters $p$ and $q$ providing optimal solutions is given by $\pi=N_{2}^{+} \xi_{2}+K v$, where $K$ is a basis matrix for ker $N_{2}$. Then, the set of all optimal state trajectories are given by (22), where the values of $p$ and $q$ are parametrized by the latter.

Problem 3 (Assigned terminal state and weighted initial state).

Since the initial state is quadratically weighted in the performance index, the Pontryagin set of equations includes a boundary condition on $\lambda(0)$, expressed by $\lambda(0)=-P_{0} x(0)$. We obtain

$$
\left(P_{0}+P_{+}\right) p+\left(P_{0}-P_{*}\right) A_{*}^{k_{f}} q=0 .
$$

Equations $x\left(k_{f}\right)=x_{f}$ and (35), in view of $(22)$ evaluated at $k=k_{f}$, leads to the compact expression

$$
\xi_{3}=N_{3} \pi \quad \text { where } \quad \xi_{3}:=\left[\begin{array}{c}
0 \\
x_{f}
\end{array}\right] \quad \text { and } \quad N_{3}:=\left[\begin{array}{cc}
P_{0}+P_{+} & \left(P_{0}-P_{*}\right) A_{*}^{k_{f}} \\
A_{+}^{k_{f}} & I
\end{array}\right] .
$$

Let $K$ is a basis matrix for ker $N_{3}$. Again, $p$ and $q$ can be obtained by $\pi=N_{3}^{+} \xi_{3}+K v$, since $\xi_{3} \in \operatorname{im} N_{3}$.

Problem 4 (Weighted initial and terminal states).

Relation (22) evaluated at $k=0$ and $k=k_{f}$ can be expressed in compact form as

$$
\left[\begin{array}{l}
0 \\
0
\end{array}\right]=N_{4}\left[\begin{array}{l}
p \\
q
\end{array}\right] \quad \text { where } \quad N_{4}:=\left[\begin{array}{cc}
P_{0}+P_{+} & \left(P_{0}-P_{*}\right) A_{*}^{k_{f}} \\
\left(P_{f}-P_{+}\right) A_{+}^{k_{f}} & P_{f}+P_{*}
\end{array}\right] .
$$

Let $K$ be a basis matrix for ker $N_{4}$. Clearly, $p$ and $q$ can be obtained by $\pi=K v$.

\subsection{Optimal cost}

In this section, we present general expressions for the optimal value of the cost function corresponding to the four optimal control problems described in Section 2. First we express such value as a quadratic forms in $\pi:=\left[\begin{array}{ll}p^{\top} & q^{\top}\end{array}\right]^{\top}$.

Lemma 1 The following identities hold.

$$
\begin{aligned}
& P_{+}-A_{+}^{\top} P_{+} A_{+}-Q+S K_{+}+K_{+}^{\top} S^{\top}-K_{+}^{\top} R K_{+}=0, \\
& P_{*}-A_{*}^{\top}, P_{*} A_{*}-A_{*}^{\top} Q A_{*}+A_{*}^{\top} S K_{*}+K_{*}^{\top} S^{\top} A_{*}-K_{*}^{\top} R K_{*}=0, \\
& A_{+}^{\top} P_{*}-P_{*}-Q A_{*}+S K_{*}+K_{+}^{\top} S^{\top} A_{*}-K_{+}^{\top} R K_{*}=0 .
\end{aligned}
$$


Proof: The first equality reduces to the $\operatorname{DARE}(\Sigma)$ written with respect to $P_{+}$by virtue of $(8)$. The second and the third identities follow from (24), (26) and (28).

Theorem 6 Consider Problem $i, i \in\{1,2,3,4\}$. The optimal cost is $J^{o}=\pi^{\top} V_{i} \pi$, where

$$
\begin{aligned}
& V_{1}:= {\left[\begin{array}{ll}
P_{+}-\left(A_{+}^{\top}\right)^{k_{f}} P_{+} A_{+}^{k_{f}} & \left(A_{+}^{\top}\right)^{k_{f}} P_{*}-P_{*} A_{*}^{k_{f}} \\
\left(A_{*}^{\top}\right)^{k_{f}} P_{+}-P_{+} A_{+}^{k_{f}} & P_{*}-\left(A_{*}^{\top}\right)^{k_{f}} P_{*} A_{*}^{k_{f}}
\end{array}\right], } \\
& V_{2}:=\left[\begin{array}{ll}
P_{+}+\left(A_{+}^{\top}\right)^{k_{f}}\left(P_{f}-P_{+}\right) A_{+}^{k_{f}} & \left(A_{+}^{\top}\right)^{k_{f}}\left(P_{f}+P_{*}\right)-P_{*} A_{*}^{k_{f}} \\
\left(P_{f}-P_{+}\right) A_{+}^{k_{f}}+\left(A_{*}^{\top}\right)^{k_{f}} P_{+} & \left(P_{f}+P_{*}\right)-\left(A_{*}^{\top}\right)^{k_{f}} P_{*} A_{*}^{k_{f}}
\end{array}\right], \\
& V_{3}:=\left[\begin{array}{ll}
\left(P_{+}+P_{0}\right)-\left(A_{+}^{\top}\right)^{k_{f}} P_{+} A_{+}^{k_{f}} & \left(A_{+}^{\top}\right)^{k_{f}} P_{*}+\left(P_{0}-P_{*}\right) A_{*}^{k_{f}} \\
\left(A_{*}^{\top}\right)^{k_{f}}\left(P_{+}+P_{0}\right)-P_{+} A_{+}^{k_{f}} & P_{*}+\left(A_{*}^{\top}\right)^{k_{f}}\left(P_{0}-P_{*}\right) A_{*}^{k_{f}}
\end{array}\right], \\
& V_{4}:=0 .
\end{aligned}
$$

Proof: Consider Problem 1. From (2) and (22), the optimal cost can be written as

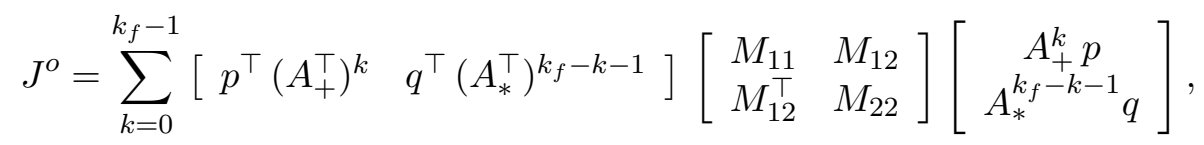

with

$$
\begin{aligned}
& M_{11}:=Q-S K_{+}-K_{+}^{\top} S^{\top}+K_{+}^{\top} R K_{+}=P_{+}-A_{+}^{\top} P_{+} A_{+}, \\
& M_{12}:=Q A_{*}-S K_{*}-K_{+}^{\top} S^{\top} A_{*}+K_{+}^{\top} R K_{*}=A_{+}^{\top} P_{*}-P_{*} A_{*}, \\
& M_{22}:=A_{*}^{\top} Q A_{*}-A_{*}^{\top} S K_{*}-K_{*}^{\top} S^{\top} A_{*}+K_{*}^{\top} R K_{*}=P_{*}-A_{*}^{\top} P_{*} A_{*},
\end{aligned}
$$

where the equalities follow from Lemma 1 . Notice that

$$
\begin{aligned}
& \sum_{k=0}^{k_{f}-1}\left(A_{+}^{\top}\right)^{k}\left(P_{+}-A_{+}^{\top} P_{+} A_{+}\right) A_{+}^{k}=P_{+}-\left(A_{+}^{\top}\right)^{k_{f}} P_{+} A_{+}^{k_{f}}, \\
& \sum_{k=0}^{k_{f}-1}\left(A_{+}^{\top}\right)^{k}\left(A_{+}^{\top} P_{*}-P_{*} A_{*}\right) A_{*}^{k_{f}-k-1}=\left(A_{+}^{\top}\right)^{k_{f}} P_{*}-P_{*} A_{*}^{k_{f}}, \\
& \sum_{k=0}^{k_{f}-1}\left(A_{*}^{\top}\right)^{k_{f}-k-1}\left(P_{*}-A_{*}^{\top} P_{*} A_{*}\right) A_{*}^{k_{f}-k-1}=P_{*}-\left(A_{*}^{\top}\right)^{k_{f}} P_{*} A_{*}^{k_{f}},
\end{aligned}
$$

that, by substitution, yield (41). Consider Problem 2. By virtue of (22) evaluated at $k=k_{f}$, the quadratic form $x^{\top}\left(k_{f}\right) P_{f} x\left(k_{f}\right)$ is summed by (41) to obtain (42). Consider Problem 3. By virtue of $(22)$ evaluated at $k=0$, the quadratic form $x^{\top}(0) P_{0} x(0)$ is summed by (41) to obtain (43).

Alternative expressions of the optimal cost are provided as a quadratic form in the assigned states.

Corollary 1 The following results hold.

- Consider Problem 1. Let $N_{1}$ be defined in (32) and $V_{1}$ be defined in (41). Let $x:=\left[\begin{array}{ll}x_{0}^{\top} & x_{f}^{\top}\end{array}\right]^{\top}$. The optimal cost is $J^{o}=x^{\top} N_{1}^{-\top} V_{1} N_{1}^{-1} x$.

- Consider Problem 2. Let $M \in \mathbb{R}^{2 n \times n}$ be such that $\pi=M x_{0}$. Let $V_{2}$ be defined in (42). The optimal cost is $J^{o}=x_{0}^{\top} M^{\top} V_{2} M x_{0}$.

- Consider Problem 3. Let $N \in \mathbb{R}^{2 n \times n}$ be such that $\pi=N x_{f}$. Let $V_{3}$ be defined in (43). The optimal cost is $J^{o}=x_{f}^{\top} N^{\top} V_{3} N x_{f}$. 


\section{An example and concluding remarks}

\subsection{An example}

The formulas that provide the optimal value of the cost can be exploited to solve non-standard optimal control problems. For example, consider the case when $x(0)=x_{0}$ and the optimal cost to be minimized is

$$
J(x, u)=\left(x\left(k_{f}\right)-\xi_{f}\right)^{\top} P_{f}\left(x\left(k_{f}\right)-\xi_{f}\right)+\sum_{k=0}^{k_{f}-1}\left[x^{\top}(k) u^{\top}(k)\right]\left[\begin{array}{cc}
Q & S \\
S^{\top} & R
\end{array}\right]\left[\begin{array}{l}
x(k) \\
u(k)
\end{array}\right],
$$

where $\xi_{f} \in \mathbb{R}^{n}$ is given. This non-standard LQ problem can be tackled as follows. First, determine the minimum of the sum in $(45)$ with $x(0)=x_{0}$ and $x\left(k_{f}\right)=\bar{x}_{f}$. Such minimum is a quadratic form in $x_{0}$ and $\bar{x}_{f}$ (see Theorem 6 and Corollary 1 ):

$$
\begin{aligned}
J^{\prime}\left(x_{0}, \bar{x}_{f}\right) & =\min _{x\left(k_{f}\right)=\bar{x}_{f}} \sum_{k=0}^{k_{f}-1}\left[\begin{array}{ll}
x^{\top}(k) & u^{\top}(k)
\end{array}\right]\left[\begin{array}{cc}
Q & S \\
S^{\top} & R
\end{array}\right]\left[\begin{array}{l}
x(k) \\
u(k)
\end{array}\right] \\
& =\left[\begin{array}{ll}
x_{0}^{\top} & \bar{x}_{f}^{\top}
\end{array}\right] N_{1}^{-\top} V_{1} N_{1}^{-1}\left[\begin{array}{l}
x_{0} \\
\bar{x}_{f}
\end{array}\right]
\end{aligned}
$$

where $N_{1}$ is defined in (32) and $V_{1}$ is defined in (41). Now, the minimum of $J(x, u)$ can be attained by performing a minimization over $\bar{x}_{f}$ :

$$
\min J(x, u)=\min _{\bar{x}_{f}}\left[\left(\bar{x}_{f}-\xi_{f}\right)^{\top} P_{f}\left(\bar{x}_{f}-\xi_{f}\right)+J^{\prime}\left(x_{0}, \bar{x}_{f}\right)\right] .
$$

Therefore, the problem reduces to the minimization of a static quadratic function in $\bar{x}_{f}$, which is standard and easy to solve. Once $\bar{x}_{f}$ is determined, $p$ and $q$ are computed by inversion of equation (32), and (22) provides the optimal control. To the best of the authors' knowledge, this problem has not been solved (and is not easy to solve) with the classical approach to the LQ problem.

\subsection{Concluding remarks}

A novel approach has been presented for the solution of a class of finite-horizon linear quadratic problems in the discrete-time case. It has been shown that the set of solutions of the extended symplectic system, which represents necessary conditions for optimality, can be parametrized in closed-form in terms of two vectors $p$ and $q$. Their values can be determined by imposing the boundary conditions corresponding to the particular problem considered.

This procedure is particularly convenient since it yields reliable software algorithms for the solution of all the optimal control problems considered herein: in fact, the result presented in Theorem 5 involves the stabilizing solution of a discrete-time algebraic Riccati equation and the solution of a Stein equation, that may be computed by standard and robust algorithms available in any control package (see the MATLAB ${ }^{\circledR}$ routines dare.m and dlyap.m). Furthermore, the expression of the optimal control is given in terms of powers of strictly stable matrices in the overall time interval, thus ensuring that the solution proposed is robust even for large timehorizons.

\section{References}

[1] B.D.O. Anderson and J.B. Moore. Optimal Control: Linear Quadratic Methods. Prentice Hall International, London, 1989. 
[2] F. Blanchini and F.A. Pellegrino. Relatively optimal control and its linear implementation. IEEE Transactions on Automatic Control, AC-48:2151-2162, 2003.

[3] D.J. Clements and B.D.O. Anderson. Singular optimal control: the linear quadratic problem. In A.V. Balakrishnan and M. Thoma, editors, Lecture notes in control and information sciences, volume 5, page 93. Springer-Verlag, New York, 1978.

[4] A. Ferrante and B.C. Levy. Canonical form for symplectic matrix pencils. Linear Algebra and Its Applications, 274:259-300, 1998.

[5] A. Ferrante, G. Marro, and L. Ntogramatzidis. Employing the algebraic Riccati equation for the solution of the finite-horizon LQ problem. In Proceedings of the 42th IEEE Conference on Decision and Control (CDC 2003), Hgatt Regency Maui, Hawaii, USA, December 2003.

[6] V. Ionescu, C. Oară, and M. Weiss. Generalized Riccati theory and robust control, a Popov function approach. Wiley, 1999.

[7] E. Kondo, T. Kiyota, and S. Sunaga. Closed-loop expressions of fixed-end-point LQ optimal control for digital systems. In Proceedings of the 12th IFAC Congress, volume 1, pages 173178, July 1993.

[8] H. Kwakernaak and R. Sivan. Linear Optimal Control Systems. John Wiley \& Sons, New York, 1972.

[9] P. Lancaster and L. Rodman. Algebraic Riccati equations. Clarendon Press, Oxford, 1995.

[10] B.C. Levy and A. Ferrante. Characterization of stationary discrete-time gaussian reciprocal processes over a finite interval. SIAM Journal of matrix analysis and applications, $24(2): 334-355,2002$.

[11] F.L. Lewis and V. Syrmos. Optimal Control. John Wiley \& Sons, New York, 1995.

[12] G. Marro, D. Prattichizzo, and E. Zattoni. A geometric insight into the discrete time cheap and singular LQR problems. IEEE Transactions on Automatic Control, 47(1):102-107, 2002 .

[13] B.P. Molinari. The time-invariant linear-quadratic optimal control problem. Automatica, 13:347-357, 1977.

[14] L. Ntogramatzidis. A simple solution to the finite-horizon LQ problem with zero terminal state. Kybernetika, 39(4):483-492, 2003.

[15] C. Oară and P. Van Dooren. An improved algorithm for the computation of structural invariants of a system pencil and related geometric aspects. Systems $\&$ Control Letters, 30:39-48, 1997.

[16] A.C.M. Ran and H.L. Trentelman. Linear quadratic problems with indefinite cost for discrete time systems. SIAM Journal of matrix analysis and applications, 14(3):776-797, 1993.

[17] A. Saberi, P. Sannuti, and B.M. Chen. $\mathrm{H}_{2}$ Optimal Control. System and Control Engineering. Prentice Hall International, London, 1995. 